\title{
BMJ Open Prognostic factors in low back pain individuals undergoing steroid and anaesthetic intra-articular facet joint infiltration: a protocol for a prospective, longitudinal, cohort study
}

\author{
João Carlos Rodrigues, ${ }^{\oplus 1,2}$ Arthur Werner Poetscher, ${ }^{\circ}{ }^{3}$ Mario Lenza, ${ }^{3}$ \\ Alberto Ofenhejm Gotfryd, ${ }^{3}$ Délio Eulálio Martins Filho, ${ }^{\circ} 3$ \\ Luciano Miller Reis Rodrigues, ${ }^{\oplus 3}$ Rodrigo Gobbo Garcia, ${ }^{\oplus}$ \\ Laercio Alberto Rosemberg, ${ }^{1,2}$ Durval do Carmo Santos Barros, ${ }^{\circledR 1}$ \\ Eduardo Noda Kihara Filho, ${ }^{1}$ Mario Ferretti, ${ }^{3}$ Gilbert Sung Soo Bang ${ }^{3}$
}

To cite: Rodrigues JC,

Poetscher AW, Lenza M, et al. Prognostic factors in low back pain individuals undergoing steroid and anaesthetic intra-articular facet joint infiltration: a protocol for a prospective, longitudinal, cohort study. BMJ Open 2019;9:e026903. doi:10.1136/ bmjopen-2018-026903

- Prepublication history for this paper is available online. To view these files please visit the journal online (http://dx.do org/10.1136/bmjopen-2018026903).

Received 25 September 2018 Revised 15 April 2019 Accepted 30 May 2019
Check for updates

(C) Author(s) (or their employer(s)) 2019. Re-use permitted under CC BY-NC. No commercial re-use. See rights and permissions. Published by BMJ.

For numbered affiliations see end of article.

Correspondence to MD João Carlos Rodrigues; joao.rodrigues@einstein.br

\section{ABSTRACT}

Introduction Lumbar pain of facet origin is a common problem worldwide. For those patients not responding to traditional treatment, one approach may be intra-articular infiltration of corticoid and anaesthetic. However, despite the increasing demand for this procedure, no consensus exists regarding its therapeutic value. The selection of eligible participants may be a determining factor since only those with an inflammatory process will benefit from the use of corticosteroids. This study aims to identify differences in disability, pain and quality of life scores in individuals with and without facet joint inflammation who were diagnosed using MRI.

Method and analysis This prospective cohort will include individuals older than 18 years with a clinical diagnosis of facet syndrome who underwent intra-articular infiltration. Changes in scores of pain, disability and quality of life questionnaires at 1, 3, 6 and 12 months of follow-up compared with baseline will be analysed. An MRI examination performed before infiltration will help to distinguish between exposed (with inflammation) and nonexposed (non-inflammation) groups with facet syndrome. The primary outcome will be the disability questionnaire (Roland Morris), and the secondary outcomes will be the score questionnaires for pain (Visual Analogue Scale), quality of life (EuroQol Quality of Life Questionnaire) and disability (Oswestry).

Ethics and dissemination The Internal Review Board approved this study, which started only after the approval number (5291417.0.0000.0071) was received. All recruited participants will receive a verbal explanation about the purpose of the study, and their decision to participate will be free and voluntary. All participants enrolled in the study will provide a signed informed consent form including confidentiality terms. The results obtained in this study will be presented at national and international conferences and published in peer-reviewed scientific journals to disseminate the knowledge.

Trials registration number NCT03304730; Pre-results.
Strengths and limitations of this study

This is the first study to test inflammatory markers of facet inflammation based on the results of MRI examinations as prognostic factors of intra-articular facet infiltration.

- This evaluation is not restricted to exposure only (with or without facet inflammation); demographics and facet morphological characteristics will also be assessed to determine their influence on the outcomes.

- Limitations of this study are related to the selection and follow-up bias of the participants. Investigators will closely monitor the study and take all possible actions to avoid these factors.

\section{INTRODUCTION}

Low back pain (LBP) is one of the significant health problems worldwide, presenting a variable point prevalence among several countries estimated at $33 \%$ in Belgium, $28.4 \%$ in Canada, $14 \%$ in the UK, $13.7 \%$ in Denmark, $12 \%$ in Sweden and $6.8 \%$ in North America. ${ }^{1}$ Studies using lumbar spine imaging methods have reported that facet osteoarthritis is one of the most prevalent conditions among individuals with LBP, affecting $59.6 \%$ of men and $66.7 \%$ of women. ${ }^{2}$

Several treatments are used in LBP secondary to facet origin. The most common treatments are non-steroidal anti-inflammatory drugs provided orally and physical rehabilitation. ${ }^{34}$ Interventional procedures, such as intra-articular facet infiltration (IFI) or periarticular facet infiltration for neural medial branch block and radiofrequency denervation (RFD), are considered minimally 
invasive options and are indicated for cases in which traditional treatment fails. ${ }^{5}$ Another option is arthrodesis surgery. ${ }^{6}$

IFI has been used as a treatment option because it increases the concentration of steroids in the facet joint without the undesirable side effects associated with systemic administration of steroids. ${ }^{7}$ Even though many publications are available, the treatment of persistent LBP remains controversial. ${ }^{8-13}$ Two recent feasibility studies for a randomised controlled trial (RCT) found in their survey of the literature that the interpretations given to systematic reviews about IFI were usually inappropriate. ${ }^{1415}$ The lack of high-quality evidence in these systematic reviews does not corroborate the use of IFI in practice, notwithstanding some moderate-quality trials revealed the effectiveness of the treatment. A recent high-quality systematic review has carefully condensed the IFI current evidence in their conclusion: "The studies found here were clinically diverse and precluded any meta-analysis. Many methodological issues were identified. The positive results, while interpreted with caution, do suggest that there is a need for further high-quality work in this area." 16 The lack of substantial evidence led the National Institute for Health and Care Excellence (NICE) and the American Pain Society not to support the use of IFI. ${ }^{17}{ }^{18}$ Instead, the last NICE update, revised in 2016, recommended the use of RFD after the medial nerve branch diagnostic block confirms facet-related pain. ${ }^{18}$ While most reviews conclude that RFD is effective and IFI is not, others dispute this. ${ }^{13}{ }^{19-22}$ In comparing the efficacy between IFI and RFD, some studies show divergent results. A systematic review and meta-analysis including nine studies on the overall quality of evidence rated low to moderate reported that RFD is more effective than placebo in pain control and functional improvement and that it may also be more effective than steroid injections in pain control. ${ }^{21} \mathrm{~A}$ double-blind RCT reported that facet-related LBP could be treated with IFI or RFD with appropriate pain relief and functional improvement for at least 6 months, with no differences between treatments. ${ }^{20}$ Despite the controversy and lack of guidelines supporting IFI, this procedure is still one of the most used treatments, with growing demand in recent decades. ${ }^{23} 24$

The appropriate selection of participants seems to be a determining factor in the outcome of IFI given that, in theory, only those with ongoing inflammation would benefit from steroid infiltration. The identification of these individuals poses a challenge because the clinical diagnosis of facet origin pain is often imprecise and diagnostic injection is limited. ${ }^{25}{ }^{26}$ The clinical diagnosis of facet-related pain has limited evidence, and studies do not defend the use of some symptoms or exasperating characteristics as representative of a disorder in the facet joint. ${ }^{27-29}$ The best current practice in identifying facet-related pain has some validity when paraspinal pain and a regular compression pattern of combined motion are definite. ${ }^{30-34}$ Diagnostic injection with lidocaine has been the method of choice to define whether a given patient has facet-related pain; however, many criticisms have emerged about the validity of this pretest. ${ }^{26}$ The use of any pretest (uncontrolled, anaesthetic-controlled or placebo-controlled) can constitute a bias and impair the external validity of the study. Diagnostic injection may modify the patients' baseline by increasing or decreasing their sensitivity to IFI. The results obtained cannot be extrapolated to the target population, and the impact of IFI is limited to patients who have previously undergone a pretest. A study of bone scintigraphy with single photon emission CT (SPECT) identified individuals with LBP of facet origin who would benefit from steroid IFI. This study reported that participants with positive SPECT facet uptake had better pain scores in the short-term follow-up than those individuals with negative SPECT facet uptake. ${ }^{35}$ MRI helps to identify the presence (or absence) of inflammation in the facet joint which is usually suggested by the presence of bone oedema, periarticular soft tissue oedema, joint effusion and synovitis. ${ }^{36}$ Although lumbar spine MRI has limitations in the assessment of LBP, when confronted with a patient who has failed conservative treatment, the classic course has been to perform lumbar spine MRI rather than SPECT. Thus, a proper and accurate diagnosis of facet inflammatory findings obtained by MRI will help identify individuals expected to benefit from IFI. To our knowledge, no inflammatory markers of facet inflammation based on MRI examination have been tested as prognostic factors of IFI efficacy.

\section{HYPOTHESIS AND OBJECTIVES}

We hypothesise that MRI facet findings are prognostic factors of IFI efficacy.

The primary objective of this study is to determine prognostic factors in individuals with LBP who have undergone IFI treatment and to identify differences in disability, pain and quality of life scores between participants with and without facet joint inflammation detected by MRI examination.

The secondary objective of the study is to determine the correlation between age, sex, formal education, body mass index (BMI) and professional activity and pain, disability and quality of life outcomes.

\section{METHOD AND ANALYSIS}

This study protocol follows the guidelines of the Strengthening the Reporting of Observational Studies in Epidemiology. ${ }^{37}$

\section{Study design}

Single-centre prospective longitudinal cohort.

\section{Study setting}

The radiology department, the image-guided intervention centre and the outpatient spine clinic of a tertiary 
hospital in the city of São Paulo, Brazil, conducted this study in collaboration.

\section{Participants}

\section{Inclusion criteria}

The following are inclusion criteria for participants:

- Older than 18 years.

- Fluent or native Portuguese language speaker.

- Continuous or intermittent LBP for at least 3 months. ${ }^{38}$

- LBP with or without irradiation to the gluteal region indicating facet syndrome (pain is aggravated by spine extension or bending towards the affected side; pain is exacerbated by prolonged sitting or walking up steps, as well as when retaining one position for a prolonged time).$^{39}$

- Failure of traditional treatment includes, but is not limited to, physical or drug therapy.

- An understanding of the purpose of the study.

- Voluntarily provision of a free and informed consent form, by themselves or through their partners, and completion of the questionnaires, before undergoing infiltration, over the telephone or online during the follow-up.

\section{Exclusion criteria}

The following are exclusion criteria for participants:

- Younger than 18 years old.

- Symptomatic lumbar spinal stenosis with claudication or radiculopathy.

- Evidence of radiculopathy.

- Active rheumatological diseases.

- Congenital or acquired deformities of the lumbar spine.

- Fracture or sequel of lumbar spine fracture of traumatic, pathological or osteoporotic origin.

- Surgical manipulation of the lumbar spine.

- MRI scans of limited quality and incomplete sequences.

- Treatment with systemic steroids less than 1 month before the IFI.

- Treatment with IFI with steroids within the last 6 months.

- Diagnosis with uncontrolled diabetes mellitus.

- History of allergy to anaesthetics or an adverse reaction to steroids.

- Pregnant women or women who breast fed.

- Inability to be contacted over the phone during follow-up.

\section{Participant selection and recruitment}

A consecutive sample of individuals referred to the image-guided intervention centre to perform IFI will be screened for eligibility. On the day of the IFI procedure, participants are admitted to the image-guided intervention centre by a trained research assistant (RA) to be interviewed about his/her willingness to participate in the study. At this time, inclusion and exclusion criteria will be applied. The RA will explain the purposes of the research and will invite those who meet the inclusion criteria to participate. All study details will be described, and the informed consent form will be read aloud. All questions about study objectives, risks, benefits and confidentiality will be answered, and individuals who agree to participate will date and sign the informed consent form. A copy of the consent form will be attached to participants' medical records, and another copy will be given to them. After signing the informed consent form and before IFI treatment, participants will provide demographic data and complete pretreatment forms. If for any reason, the participant is unable to sign the informed consent form, a verbal explanation of the study will be provided, and the participant will be asked to give verbal consent in the presence of a witness who will sign the informed consent form. Reasons for exclusion or refusal to participate will be recorded. Recruitment will last 36 months. As described in the Sample size estimation section, we expect to enrol 147 participants. figure 1 shows a flowchart depicting the study procedures.

\section{Variables}

Primary outcome

Disability

Change in disability will be quantified using the validated Brazilian version of the Roland Morris questionnaire that will be administered at recruitment and 1, 3,6 and 12 months after IFI treatment. ${ }^{40}$

\section{Secondary outcome}

Low back pain

Changes in the intensity of LBP will be assessed using the Visual Analogue Scale (VAS) and the number of analgesic tablets taken per day (an indirect marker of pain intensity) at recruitment and 1, 3, 6 and 12 months after IFI.

\section{Disability}

Changes in disability will also be quantified using the validated Brazilian version of the Oswestry Back Pain Disability Index (ODI) that will be administered at recruitment and 1, 3,6 and 12 months after IFI. ${ }^{41}$

\section{Quality of life}

Changes in quality of life will be assessed using the validated Brazilian version of the EuroQol (EQ-5D) questionnaire. This measurement tool will be administered at recruitment and 1, 3, 6 and 12 months after IFI. ${ }^{42}$ The mental status (depression and anxiety) of participants will be assessed by the questionnaire EQ-5D, which already includes a specific section for this analysis.

\section{Exposures}

The exposure of interest is signs of inflammation (synovitis, joint effusion, bone oedema, periarticular soft tissue oedema) in the facet joint lumbar spine. Data on the exposure of interest will be evaluated by MRI examination to the spine, as detailed in the item MRI and reading parameter. 


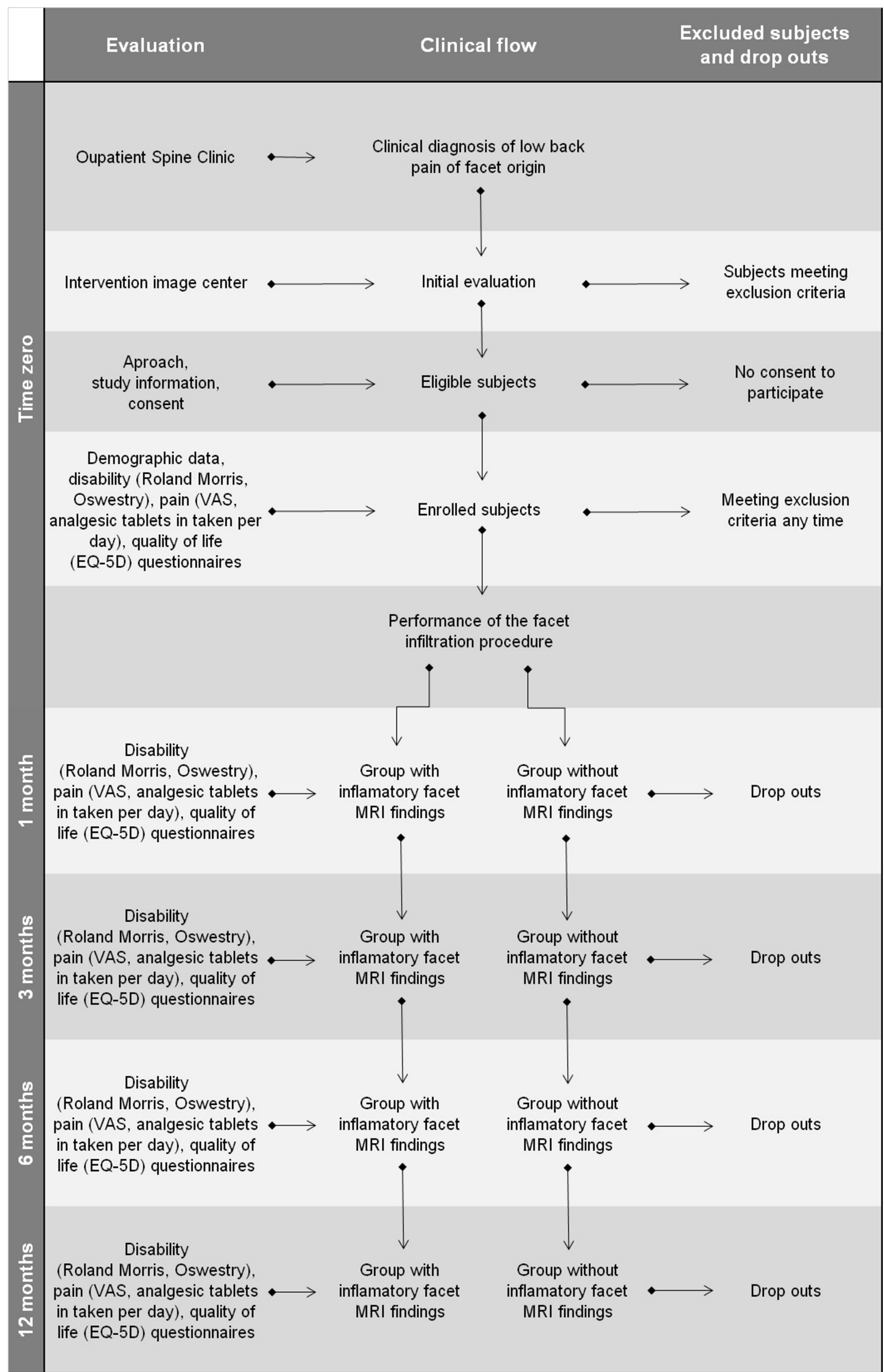

Figure 1 Clinical pathway for intra-articular facet infiltration study. EQ-5D, EuroQol Quality of Life Questionnaire; VAS, Visual Analogue Scale. 


\section{Confounding variables}

Data on the following confounding variables will be collected: age (years), sex (male or female), BMI, formal education (years of study), smoking (number of cigarettes per day), type of occupation (number of hours sitting or standing, carrying weight or not) and physical rehabilitation (number of sessions). As this information may change over time, the analysis will include data gathered at the time of the last assessment.

Most participants enrolled in this study are regularly undergoing physical therapy before and after the infiltration procedure as part of conservative treatment. In this observational study, this variable is not controlled, but it will be adequately monitored and the influence in the outcome will be analysed in both groups with or without an exposure factor.

For the anthropometric measurement, BMI, weight and height in the preprocedure room will be measured in a standardised way. Weight will be recorded in kilograms on a digital floor scale, and participants will be requested to take off their shoes and wear only underwear and an apron provided by the intervention centre. Height will be measured in centimetres using a ruler fixed to the wall, and the participant will be asked to remain in the orthostatic position without shoes.

The degree of facet osteoarthritis will be considered a possible confounding variable and will be evaluated using imaging described in the item $C T$ reading parameters.

\section{IFI procedure}

The IFI procedure will be carried out by the physician at the intervention centre in a standardised way with the conscious patients in the ventral decubitus position. The physician will infiltrate the facet level defined in the medical request form. After asepsis and antisepsis, 2\% lidocaine will be used as a local anaesthetic for sensitive blockage of the skin and subcutaneous tissue. CT images obtained from the Somatom (Siemens Medical Solutions, Erlangen, Germany) apparatus will guide the correct positioning and progression of a $22 \mathrm{G}$ needle. The use of CT is justified because high precise intra-articular injection can be achieved with extremely high procedural accuracy at an effective irradiation dose comparable with that of fluoroscopy. ${ }^{43}$ The irradiation dose used will be the minimum possible to obtain images of sufficient quality to guide the location and progression of the needle. Although MRI-guided infiltration is technically feasible and has the advantage of not using ionising radiation, this method is not commonly used and doubles the cost and time of the procedure compared with $\mathrm{CT}^{44}$ After image confirmation of the correct intra-articular positioning of the $22 \mathrm{G}$ needle, a solution containing triamcinolone $(20 \mathrm{mg} / \mathrm{mL})$ and ropivacaine $(7.5 \mathrm{mg} / \mathrm{mL})$ in a $1: 1$ ratio and $1.0 \mathrm{ml}$ volume will be injected. This type of steroid is absorbed completely but slowly, and the onset of the anti-inflammatory effect occurs after 24 hours and lasts 4 to 6 weeks. After the procedure, the participant will be discharged and advised to rest at home for 24 hours.

\section{Adverse effects}

The risks inherent to the IFI procedure, such as bleeding, infection and discomfort, among others, whether rare or common, will be detailed to participants prior to the procedure according to the standardised institutional protocol and clinical routine of the intervention centre. A specific risk form, containing information on possible complications and the use of ionising radiation, will be signed by the participant before the procedure. Any complications will be registered.

\section{Early withdrawal from the study}

Participants will be excluded from the study in cases of withdrawal of consent, death, failure to meet eligibility criteria, loss of follow-up, systemic use of steroids and the performance of another IFI treatment or lumbar spine surgery. For each excluded case, the reason and circumstances for the withdrawal will be detailed. Participants' data collected until that point will be included in the final analysis of the study.

\section{MRI examinations}

Spine MRI examinations will be conducted in a routine clinical fashion on a 1.5 T magnet HDX (GE Healthcare, Milwaukee, USA) according to the departmental protocol: participants will be scanned in a supine position and their leg extended using a multichannel spine dedicated coil. Examinations will use the following sequences: sagittal T2-weighted fat-suppressed (TR/TE, 2756/58; number of excitations (NEX), 2; matrix, 256 $\times 192$; thickness, $4 \mathrm{~mm}$; field of view (FOV), $40 \mathrm{~cm}$ ), sagittal T1-weighted $(357 / 15 ; 2 ; 320 \times 256 ; 4 \mathrm{~mm} ; 40 \mathrm{~cm})$, sagittal T2-weighted $(2890 / 58,2 ; 256 \times 192 ; 4 \mathrm{~mm} ; 40 \mathrm{~cm})$, coronal T2-weighted fat-suppressed $(2756 / 58,2 ; 256 \times 192 ; 4 \mathrm{~mm} ; 40 \mathrm{~cm})$, axial T2-weighted (2756/58, 2; 256×192; $4 \mathrm{~mm} ; 25 \mathrm{~cm})$.

\section{MRI reading parameters}

The degree of inflammation in the facet joint will be evaluated using T2-weighted fat-suppressed sequences, according to the classification system proposed by Czervionke ${ }^{36}$ as follows:

- Grade 0: no oedema abnormality.

- Grade 1: oedema confined to joint capsule.

- Grade 2: periarticular oedema involving less than $50 \%$ of the perimeter of the joint.

- Grade 3: periarticular oedema involving more than $50 \%$ of the perimeter of the joint.

- Grade 4: the characteristics of grade 3 with the extension of oedema into the intervertebral foramen, ligamentum flavum, pedicle, transverse process or vertebral body.

\section{CT reading parameters}

CT images obtained during the facet infiltration procedure will be used to evaluate the degree of facet osteoarthritis, according to Pathria ${ }^{45}$, adapted by Weishaupt $e t$ $a l^{46}$ as follows:

- Grade 0:- normal facet joint space (2-4 mm width). 
- Grade 1: narrowing of the facet joint space $(<2 \mathrm{~mm})$ and/or small osteophytes and/or mild hypertrophy of the articular process.

- Grade 2: narrowing of the facet joint space $(<2 \mathrm{~mm})$ and/or moderate osteophytes and/or moderate hypertrophy of the articular process and/or mild subarticular bone erosion.

- Grade 3: narrowing of the facet joint space $(<2 \mathrm{~mm})$ and/or large osteophytes and/or severe hypertrophy of the articular process and/or severe subarticular bone erosion and/or subchondral cysts.

\section{Imaging readers}

Two fellowship-trained musculoskeletal radiologists (one with 20 years and the other with ten years of experience) will independently read the MRI and CT images on a Picture Archiving and Communication System VUE workstation, V.12.1.5.1156 (Carestream Health, Rochester, USA) using high-resolution monitors. A second reading in consensus will solve the discordant cases.

\section{Blinding, observation bias and measurement bias}

Five groups of individuals will be involved in this study: participants, interventional physicians (IFI performers), radiologists physicians (MRI and CT observers), data collectors (RA) and data analysts (statistician). The following actions will be taken to avoid observation bias. Researchers will not inform participants about the group to which they belong. The interventional physician will perform the IFI blinded to the MRI or questionnaire results. The radiologists will read the MRI and CT examinations blinded to the questionnaires; in addition, they will not perform IFI. The RA will collect questionnaires blinded to the MRI results or group divisions. Researchers will blind the data analysts by labelling the groups with non-identifying terms (such as A and B). The method of outcome evaluation will be identical for the exposed and unexposed groups to avoid the risk of measurement bias.

\section{Patient and public involvement}

Neither the patients nor the public were involved in planning or developing this protocol. Patients will receive information about the knowledge obtained in this study through posts or emails. Open lectures will be promoted in the hospital auditorium to disseminate the results to the public.

\section{Statistical methods}

Participants' characteristics, CT, MRI findings and scores of the pain VAS, disability (Roland Morris and Oswestry) and quality of life (EQ-5D) questionnaires will be described by means of absolute frequencies and percentages in categorical variables, or by means, SD or medians and quartiles, in addition to minimum and maximum values, in quantitative variables.

The inter-rater analysis of the CT and MRI readings will be evaluated using appropriate correlation coefficients, according to the observed distribution of many cases, through two-way tables with the percentages calculated on the total of evaluations, to obtain the proportion of agreement and disagreement between the two evaluators. We intend to measure the degree of agreement by considering two raters on a multiple scale level rating and ordinal weighting, with a coefficient such as a Percentage Agreement, Gwet's AC2, Cohen's Kappa or the Brennan-Prediger. Mixed linear models or generalised linear models in multiple approaches will be used to evaluate CT (degree of facet osteoarthritis) and MRI (degree of facet inflammation) findings and participants' characteristics (age, sex, smoking, BMI, education, occupation, physical rehabilitation sessions and analgesic intake) in the evolution of the pain (VAS), disability (RM and ODI) and quality of life (EuroQol) 1, 3, 6 and 12 months after infiltration. These models are appropriate for measurements obtained from the same participant at different times. Effect estimates will present the results (change in mean pain or means of functional disability and quality of life) with $95 \%$ CIs.

The statistical package $\mathrm{R}$ will perform the analyses considering a level of $5 \%$ as significant.

\section{Sample size estimation}

Considering the null hypothesis of no difference between groups with and without inflammation regarding the improvements in Roland Morris Questionnaire scores after 1, 3, 6 and 12 months from baseline, we assume for sample size calculations: a SD of 4.2 points, ${ }^{20}$ a significance level of 5\%, a two-sided two-sample equal-variance t-test and $95 \%$ power to detect a minimum difference of 3.1 points between improvements of the groups. The full sample size estimated is 117 , expecting that inflammation will be present in $30 \%$ of participants, which will result in a 1:2.33 ratio between inflammation and control groups. Accounting for a possible loss of $20 \%$ during follow-up, we intend to include 147 participants in the study. Calculations will use PASS 14 (2015) software (NCSS, Utah, USA).

Once the study has reached a 3-month follow-up of 70 participants, the sample size needed to compare groups with and without inflammation will be recalculated based on both the SD and the proportion of inflammation observed in this study.

\section{ETHICS AND DISSEMINATION}

This study was based on the recommendations established by the Declaration of Helsinki (2013) and Document Guidelines for the Americas. All recruited individuals will receive a verbal explanation about the purpose of this study, and participation is free and voluntary. Participants will be enrolled in the study only after signing the informed consent form, including confidentiality terms. The results obtained in this study will be presented at national and international conferences and published in peer-reviewed scientific journals to disseminate the knowledge. 


\section{DISCUSSION}

The treatment of LBP, which is associated with questionable results, has a substantial economic impact on the health system and represents a growing concern for public and medical policy-makers. ${ }^{47}$ The lack of diagnostic accuracy and the existence of various types of treatment without evidence of appropriate effectiveness are considered the leading causes of the increasing costs associated with LBP treatment. The pain of facet origin is estimated to be responsible for $30 \%$ of cases of LBP. ${ }^{48}$

Two significant inquiries about IFI remain essential research questions. One is whether the procedure is effective, and the other is who can benefit. An RCT is the most rigorous way of investigating the efficacy of IFI. However, this method could not be used to identify facet inflammation as an exposure factor. We planned this study using a cohort design because this is the available scientific method to measure the effects of a suspected exposure factor. A cohort study has limitations, as any observational research method, and such an approach may only offer clues, rather than definitive evidence of links between facet inflammation and IFI outcomes. If this cohort has a positive result, although it presents weak evidence, it can open a new approach for clinical trials to produce high-quality evidence to assess the efficiency of different treatments for LBP patients with or without facet inflammation. Facet-related pain is an intricate event, and IFI definitively would not be able to solve the issue. Perhaps, for those who respond to the IFI, the benefit will be a short-term relief which can contribute to patient adherence to rehabilitation programmes.

\section{Strengths}

To our knowledge, this is the first study to test inflammatory markers of facet inflammation based on MRI examinations as prognostic factors of IFI. This evaluation is not restricted to exposure only (with or without facet inflammation) or demographics, and the degree of facet osteoarthritis will also be assessed to determine its influence on the outcomes.

\section{Limitations}

The selection of participants is a complex process, and in this study, it is based on clinical diagnosis. Although imprecise, it is the best way to conduct the selection process because the main objective is to identify predictive factors of good response from the image point of view. The use of a pretest involving a diagnostic injection of lidocaine would be the other way to select the participants. However, this method would impair the external validity of this study. A biased sample is likely to be selected because this study requires a 1-year commitment from the participants. Many participants contacted in the recruitment phase may wish to participate but will not be able to return four times to the hospital to answer the questionnaires. A loss to follow-up bias may also occur because the exposed group is expected to have more pronounced relief of the symptoms which may discourage individuals from participating until the end of the follow-up. Investigators will enrol individuals who are easy to track and will adopt questionnaires that can be completed at the participant's homes, over the phone or online to minimise these effects. Such actions will possibly maintain the interest of those participating and will make them feel that the study is essential regardless of the IFI result.

\section{Author affiliations}

${ }^{1}$ Departamento de Radiologia, Hospital Israelita Albert Einstein, São Paulo, Brazil ${ }^{2}$ Departamento de Radiologia, Universidade de São Paulo, Hospital das Clinicas da Faculdade de Medicina, Instituto de Ortopedia e Traumatologia, São Paulo, Brazil ${ }^{3}$ Programa Locomotor, Hospital Israelita Albert Einstein, São Paulo, Brazil ${ }^{4}$ Departamento de Radiologia Intervencionista, Hospital Israelita Albert Einstein, São Paulo, Brazil

Acknowledgements The authors gratefully acknowledge the contribution of Aline Pacífico Rodrigues and Gilberto Szarf for providing all the support to turn an idea into a real project; Elivane da Silva Victor and Ana Carolina Cintra Nunes Mafra for the valuable contribution in the statistical plane; Fernanda Marques Abatepaulo, Milena Rodrigues da Fonseca, Simone Andrade Kim, Rebeca Barqueiro de Oliveira, Edvaldo Santos and Luciana Pereira de Magalhães Machado for all help with administrative issues, preparation and organization of the questionnaires; José Belem de Oliveira Neto for editing this paper for the proper English language, grammar, punctuation, spelling and overall style.

Contributors JCR conceived the study, participated in its design and coordination, and drafted the manuscript. AWP and ML participated in the conception and design of the project and writing the paper. AG, DEMF and LMRR participated in the design and coordination of the study and contributed to the critical revision of the article. RGG, DCSB, LAR, ENKF, MF and GSSB participated in the design and critical review of the manuscript. All authors read and approved the final version of the paper.

Funding The authors have not declared a specific grant for this research from any funding agency in the public, commercial or not-for-profit sectors.

Competing interests None declared.

Patient consent for publication Not required.

Ethics approval This study was approved by the Internal Board Review of our institution and the Research Ethics Committee (register number 5291417.0.0000.0071).

Provenance and peer review Not commissioned; externally peer reviewed.

Open access This is an open access article distributed in accordance with the Creative Commons Attribution Non Commercial (CC BY-NC 4.0) license, which permits others to distribute, remix, adapt, build upon this work non-commercially, and license their derivative works on different terms, provided the original work is properly cited, appropriate credit is given, any changes made indicated, and the use is non-commercial. See: http://creativecommons.org/licenses/by-nc/4.0/.

\section{REFERENCES}

1. Loney PL, Stratford PW. The prevalence of low back pain in adults: a methodological review of the literature. Phys Ther 1999;79:384-96.

2. Kalichman L, Li L, Kim DH, et al. Facet joint osteoarthritis and low back pain in the community-based population. Spine 2008;33:2560-5.

3. Chou R, Huffman LH. American Pain SocietyAmerican College of Physicians. Nonpharmacologic therapies for acute and chronic low back pain: a review of the evidence for an American Pain Society/ American College of Physicians clinical practice guideline. Ann Intern Med 2007;147:492-504.

4. Lewis A, Morris ME, Walsh C. Are physiotherapy exercises effective in reducing chronic low back pain? Phys Ther Rev 2008;13:37-44.

5. Manchikanti L, Helm S, Singh V, et al. An algorithmic approach for clinical management of chronic spinal pain. Pain Physician 2009;12:E225-64.

6. Tessitore E, Molliqaj G, Schatlo B, et al. Clinical evaluation and surgical decision making for patients with lumbar discogenic pain and facet syndrome. Eur J Radiol 2015;84:765-70.

7. Kendall PH. The side-effects of corticosteroids. Rheumatology 1964;7:253-7. 
8. Bogduk N. A narrative review of intra-articular corticosteroid injections for low back pain. Pain Med 2005;6:287-96.

9. Ribeiro LH, Furtado RN, Konai MS, et al. Effect of facet joint injection versus systemic steroids in low back pain: a randomized controlled trial. Spine 2013;38:1995-2002.

10. Falco FJ, Manchikanti L, Datta S, et al. An update of the effectiveness of therapeutic lumbar facet joint interventions. Pain Physician 2012;15:E909-53

11. Fotiadou A, Wojcik A, Shaju A. Management of low back pain with facet joint injections and nerve root blocks under computed tomography guidance. A prospective study. Skeletal Radiol 2012;41:1081-5.

12. Freyhardt $\mathrm{P}, \mathrm{H}$ Hartwig $\mathrm{T}$, De Bucourt $\mathrm{M}$, et al. MR-guided facet joint injection therapy using an open 1.0-T MRI system: an outcome study. Eur Radiol 2013;23:3296-303.

13. Manchikanti L, Hirsch JA, Falco FJ, et al. Management of lumbar zygapophysial (facet) joint pain. World J Orthop 2016;7:315.

14. Ellard DR, Underwood M, Achana F, et al. Facet joint injections for people with persistent non-specific low back pain (Facet Injection Study): a feasibility study for a randomised controlled trial. Health Technol Assess 2017;21:1-184.

15. Snidvongs S, Taylor RS, Ahmad A, et al. Facet-joint injections for non-specific low back pain: a feasibility RCT. Health Technol Assess 2017;21:1-130

16. Vekaria R, Bhatt $R$, Ellard DR, et al. Intra-articular facet joint injections for low back pain: a systematic review. Eur Spine $J$ 2016;25:1266-81.

17. Liliang PC, Lu K, Weng HC, et al. The therapeutic efficacy of sacroiliac joint blocks with triamcinolone acetonide in the treatment of sacroiliac joint dysfunction without spondyloarthropathy. Spine 2009;34:896-900.

18. NICE. Low back pain and sciatica in over 16s: assessment and management. London: NICE, 2016.

19. Juch JNS, Maas ET, Ostelo R, et al. Effect of radiofrequency denervation on pain intensity among patients with chronic lowback pain the mint randomized clinical trials. J Am Med Assoc 2017;318:68-81.

20. Lakemeier S, Lind M, Schultz W, et al. A comparison of intraarticular lumbar facet joint steroid injections and lumbar facet joint radiofrequency denervation in the treatment of low back pain: a randomized, controlled, double-blind trial. Anesth Analg 2013:117:228-35.

21. Poetscher AW, Gentil AF, Lenza M, et al. Radiofrequency Denervation for Facet Joint Low Back Pain. Spine 2014;39:E842-E849.

22. Manchikanti L, Abdi S, Atluri S, et al. An update of comprehensive evidence-based guidelines for interventional techniques in chronic spinal pain. Part II: guidance and recommendations. Pain Physician 2013;16(2 Suppl):S49-283.

23. Friedly J, Chan L, Deyo R. Increases in lumbosacral injections in the Medicare population: 1994 to 2001. Spine 2007;32:1754-60.

24. Manchikanti L, Falco FJ, Singh V, et al. Utilization of interventional techniques in managing chronic pain in the Medicare population: analysis of growth patterns from 2000 to 2011. Pain Physician 2012;15:E969-82.

25. Hancock MJ, Maher CG, Latimer J, et al. Systematic review of tests to identify the disc, SIJ or facet joint as the source of low back pain. Eur Spine J 2007;16:1539-50.

26. Bogduk N. On diagnostic blocks for lumbar zygapophysial joint pain. F1000 Med Rep 2010;2:57.

27. Laslett M, Oberg B, Aprill CN, et al. Zygapophysial joint blocks in chronic low back pain: a test of Revel's model as a screening test. BMC Musculoskelet Disord 2004;5:43.
28. Hooten WM, Martin DP, Huntoon MA. Radiofrequency neurotomy for low back pain: evidence-based procedural guidelines. Pain Med 2005;6:129-38.

29. Sharma H, Duggan A, Nazir S, et al. Setting "diagnostic reference levels" for fluoroscopy-guided spinal procedures. Orthop Proc 2012;94-B:118.

30. Depalma MJ, Ketchum JM, Trussell BS, et al. Does the location of low back pain predict its source? Pm R 2011;3:33-9.

31. Mayer TG, Robinson R, Pegues $P$, et al. Lumbar segmental rigidity: can its identification with facet injections and stretching exercises be useful? Arch Phys Med Rehabil 2000;81:1143-50.

32. Wilde VE, Ford JJ, McMeeken JM. Indicators of lumbar zygapophyseal joint pain: survey of an expert panel with the Delphi technique. Phys Ther 2007;87:1348-61.

33. Wasan AD, Jamison RN, Pham L, et al. Psychopathology predicts the outcome of medial branch blocks with corticosteroid for chronic axial low back or cervical pain: a prospective cohort study. BMC Musculoskelet Disord 2009;10:22.

34. Challinor HM, Hourigan PG, Powell R, et al. Does a regular compression combined movement test diagnose lumbar facet joint pain? Orthop Proc 2014;96-B:3.

35. Pneumaticos SG, Chatziioannou SN, Hipp JA, et al. Low back pain: prediction of short-term outcome of facet joint injection with bone scintigraphy. Radiology 2006;238:693-8.

36. Czervionke LF, Fenton DS. Fat-saturated MR imaging in the detection of inflammatory facet arthropathy (facet synovitis) in the lumbar spine. Pain Med 2008:9:400-6.

37. von Elm E, Altman DG, Egger M, et al. Strengthening the Reporting of Observational Studies in Epidemiology (STROBE) statement: guidelines for reporting observational studies. BMJ 2007;335:806-8.

38. Andersson GB. Epidemiological features of chronic low-back pain. Lancet 1999;354:581-5.

39. Daroff RB, Mazziotta JC, Jankovic J, et al. Bradley's Neurology in Clinical Practice. Elsevier Saunders 2016.

40. Nusbaum L, Natour J, Ferraz MB, et al. Translation, adaptation and validation of the Roland-Morris questionnaire--Brazil Roland-Morris. Braz J Med Biol Res 2001;34:203-10.

41. Vigatto R, Alexandre NMC, Filho HRC. Development of a Brazilian Portuguese Version of the Oswestry Disability Index. Spine 2007;32:481-6.

42. Viegas Andrade M, Noronha K, Kind P, et al. Societal Preferences for EQ-5D Health States from a Brazilian Population Survey. Value Health Reg Issues 2013;2:405-12.

43. Weininger M, Mills JC, Rumboldt Z, et al. Accuracy of CT guidance of lumbar facet joint block. AJR Am J Roentgenol 2013;200:673-6.

44. Maurer $\mathrm{MH}$, Schreiter $\mathrm{N}$, de Bucourt $\mathrm{M}$, et al. Cost comparison of nerve root infiltration of the lumbar spine under MRI and CT guidance. Eur Radiol 2013;23:1487-94.

45. Pathria M, Sartoris DJ, Resnick D. Osteoarthritis of the facet joints: accuracy of oblique radiographic assessment. Radiology 1987;164:227-30.

46. Weishaupt D, Zanetti M, Boos N, et al. MR imaging and CT in osteoarthritis of the lumbar facet joints. Skeletal Radiol 1999;28:215-9.

47. Manchikanti L, Pampati V, Falco FJ, et al. An updated assessment of utilization of interventional pain management techniques in the Medicare population: 2000 - 2013. Pain Physician 2015;18:E115-27.

48. DePalma MJ, Ketchum JM, Saullo T. What is the source of chronic low back pain and does age play a role? Pain Med 2011;12:224-33. 